\title{
Percutaneous CT fluoroscopy-guided core biopsy of pancreatic lesions: technical and clinical outcome of 104 procedures during a I 0-year period
}

Acta Radiologica

2017, Vol. 58(8) 906-913

(C) The Foundation Acta Radiologica 2016

Reprints and permissions:

sagepub.co.uk/journalsPermissions.nav DOI: $10.1177 / 0284 \mid 85$ |16678274 journals.sagepub.com/home/acr

(SAGE

\author{
Frederik Franz Strobl',*, Jens Benjamin Schwarz',*, \\ Sophia Marie Haeussler', Philipp Marius Paprottka', \\ Carsten Rist', Kolja Martin Thierfelder', Stefan Boeck ${ }^{2}$, \\ Volker Heinemann', Maximilian Ferdinand Reiser' and \\ Christoph Gregor Trumm'
}

\begin{abstract}
Background: In unclear pancreatic lesions, a tissue sample can confirm or exclude the suspected diagnosis and help to provide an optimal treatment strategy to each patient. To date only one small study reported on the outcome of computed tomography (CT) fluoroscopy-guided biopsies of the pancreas.

Purpose: To evaluate technical success and diagnostic rate of all CT fluoroscopy-guided core biopsies of the pancreas performed in a single university center during a 10 -year period.

Material and Methods: In this retrospective study we included all patients who underwent a CT fluoroscopy-guided biopsy of a pancreatic mass at our comprehensive cancer center between 2005 and 20I4. All interventions were performed under local anesthesia on a 16-row or 128-row CT scanner. Technical success and diagnostic rates as well as complications and effective patient radiation dose were analyzed.

Results: One hundred and one patients (54 women; mean age, 63.9 \pm 12.6 years) underwent a total of 104 CT fluoroscopy-guided biopsies of the pancreas. Ninety-eight of 104 interventions (94.2\%) could be performed with technical success and at least one tissue sample could be obtained. In 88 of these 98 samples, a definitive pathological diagnosis, consistent with clinical success could be achieved (89.8\%). Overall 19 minor and three major complications occurred during the intra- or 30-day post-interventional period and all other interventions could be performed without complications; there was no death attributable to the intervention.

Conclusion: CT fluoroscopy-guided biopsy of pancreatic lesions is an effective procedure characterized by a low major complication and a high diagnostic rate.
\end{abstract}

\section{Keywords}

Pancreas, biopsy, computed tomography (CT), complications, histopathology

Date received: 28 May 2016; accepted: 29 September 2016

\section{Introduction}

Pancreatic adenocarcinoma is an aggressive disease typically presenting as unresectable at the time of first diagnosis, with a median survival of 6-10 months (1). Therefore, solid pancreatic masses should be investigated carefully to exclude or confirm pancreatic malignancy (2). However, standard cross-sectional

\footnotetext{
'Institute for Clinical Radiology, Munich, Germany

${ }^{2}$ Department of Internal Medicine III, Ludwig-Maximilians-University Hospital Munich, Munich, Germany

*Equal contributors.

Corresponding author:

Frederik Franz Strobl, Ludwig-Maximilians-University Hospital, Institute for Clinical Radiology, Marchioninistr. 15, 8I377 Munich, Germany. Email: frederik.strobl@med.Imu.de
} 
imaging modalities do not always allow for a definitive diagnosis. Uncharacteristic findings make it difficult to differentiate adenocarcinoma from other causes of focal pancreatic lesions or benign inflammatory conditions (3). In such cases, histopathological analysis of a tissue sample can confirm or exclude the suspected diagnosis and help to provide an optimal treatment strategy to each patient. In addition, for an accurate planning of chemotherapy regimens, a definitive histopathological workup is necessary (4). However, it may be technically difficult to obtain tumor tissue for a pathological diagnosis before treatment initiation specifically in patients with an unresectable, locally advanced pancreatic adenocarcinoma (without distant metastases). A pre-treatment tumor biopsy is recommended by several international guidelines and medical oncologists routinely insist on a histological confirmation before initiation of chemo- or radiotherapy. This issue recently became even more important with an increase in neoadjuvant trials focusing on patients with resectable (often small tumors), borderline resectable, and locally advanced pancreatic cancer. The vast majority of these neoadjuvant studies require a confirmation of adenocarcinoma as an inclusion criterion and the inability of confirming the diagnosis by histology (or cytology) is thought to be one major barrier for trial recruitment (5).

Pancreatic fine-needle aspiration or core-needle biopsy can be performed either in an open-surgical manner or under image guidance using ultrasound, endoscopic ultrasound, or computed tomography (CT) (6). Several studies reported the results of ultrasound- or endoscopy-guided biopsies $(7,8)$. In contrast, only few studies focused on the outcome of CT-guided biopsies (8-12). Most of these studies used sequential CT guidance and not CT fluoroscopy. To the best of our knowledge only one relatively small study from 2002 covered the use of CT fluoroscopy for guidance of pancreatic true-cut biopsies (13). In contrast to sequential CT guidance, CT fluoroscopy-guided interventions can be performed faster, safer, and with a lower effective patient radiation dose $(14,15)$.

Therefore, the aim of this study was to evaluate technical success and diagnostic accuracy of all CT fluoroscopy-guided true-cut biopsies of the pancreas performed at our cancer center during a 10-year period. Additionally, complications and effective radiation dose are reported.

\section{Material and Methods}

All procedures performed in this retrospective study were in accordance with the institutional ethic committee and with the 1964 Helsinki declaration and its later amendments or comparable ethical standards. All patients gave written informed consent for the procedures of this study.

\section{Patients}

In our retrospective single-center study, all patients were included who had undergone CT fluoroscopyguided pancreatic true-cut biopsy between January 2005 and June 2014. Patients were selected by screening the RIS (Radiological Information System) for procedures encoded as "CT-guided biopsy of the pancreas." After anonymization of all patient information, data collection was performed.

This study only includes true-cut biopsies taken for histological examination. Patients who were punctured in the pancreatic region for placement of drainage were excluded from this analysis. In all cases CT, magnetic resonance imaging (MRI) and/or ultrasound had been performed prior to the intervention. A multidisciplinary team of oncologists, gastroenterologists, radiation oncologists, general surgeons, and interventional radiologists discussed clinical indication for the biopsies (e.g. suspicion of a locally advanced or metastatic pancreatic tumor by imaging criteria, neoadjuvant treatment in borderline resectable tumors). We also documented cases with former unsuccessful histological sampling of the pancreas through alternative procedures such as endoscopic ultrasound with fine needle aspiration (EUS-FNA) or endoscopic retrograde cholangiopancreatography (ERCP).

\section{Procedures}

Informed consent was obtained at least $24 \mathrm{~h}$ prior to the intervention after extensive explanation of the method and its complications. Serum creatinine level, platelet count, and coagulation values were determined prior to each intervention. Regarding laboratory cutoff values, the SIR Guidelines were followed (16).

All interventions were carried out on a 16-row or 128row scanner with CT fluoroscopy mode (10-20 mAs; $120 \mathrm{kV}$; Siemens Somatom Sensation 16, Definition AS+ or Definition Edge, Siemens Healthcare, Erlangen, Germany). For the planning and control scan we used the online dose modulation system (CareDOSE 4D, Siemens Medical Solutions, Forchheim, Germany) adapting the tube current to the patients' anatomy with the ranges of $80-120 \mathrm{kV}$ and 100-200 mAs.

Anatomical position (head, body, tail), size (diameter in $\mathrm{cm}$ ), contact of the lesion to macroscopically visible vessels (celiac artery, superior mesenteric artery [SMA] and vein, hepatic portal vein, the common hepatic artery, and the splenic artery and vein), and best approach were determined by a biphasic contrast 
enhanced planning CT including an early arterial (delay, 20s) and portal venous (delay, $70 \mathrm{~s}$ ) phase. In addition, diameter (13-18 G) and length of the true-cut biopsy system (Magnum, Bard, Murray Hill, NJ, USA) were chosen (penetration depth, 15 or $22 \mathrm{~mm}$ ). The patient was positioned depending on the planned access trajectory (supine, prone left lateral, or right lateral decubitus position).

After skin disinfection, application of $10 \mathrm{~mL}$ local anesthesia (2\% Scandicain, Astra Zeneca, London, UK) and sterile draping, the planned entry points were marked with a $22 \mathrm{G}$ fine needle used for application of the local anesthesia under CT fluoroscopy. After skin incision, the true-cut needle was consecutively inserted to the lesion under intermittent CT fluoroscopy. One to five true-cut samples were taken using a sterilizable biopsy gun (Bard Magnum). After formalin fixation these samples were sent to our local department of pathology for histological workup. Five to 10 minutes after the acquisition an unenhanced $\mathrm{CT}$ was obtained to examine the abdomen for active bleeding or other complications. Duration of the whole procedure was determined by the time period between the pre-interventional planning $\mathrm{CT}$ and the last control CT (Fig. 1).
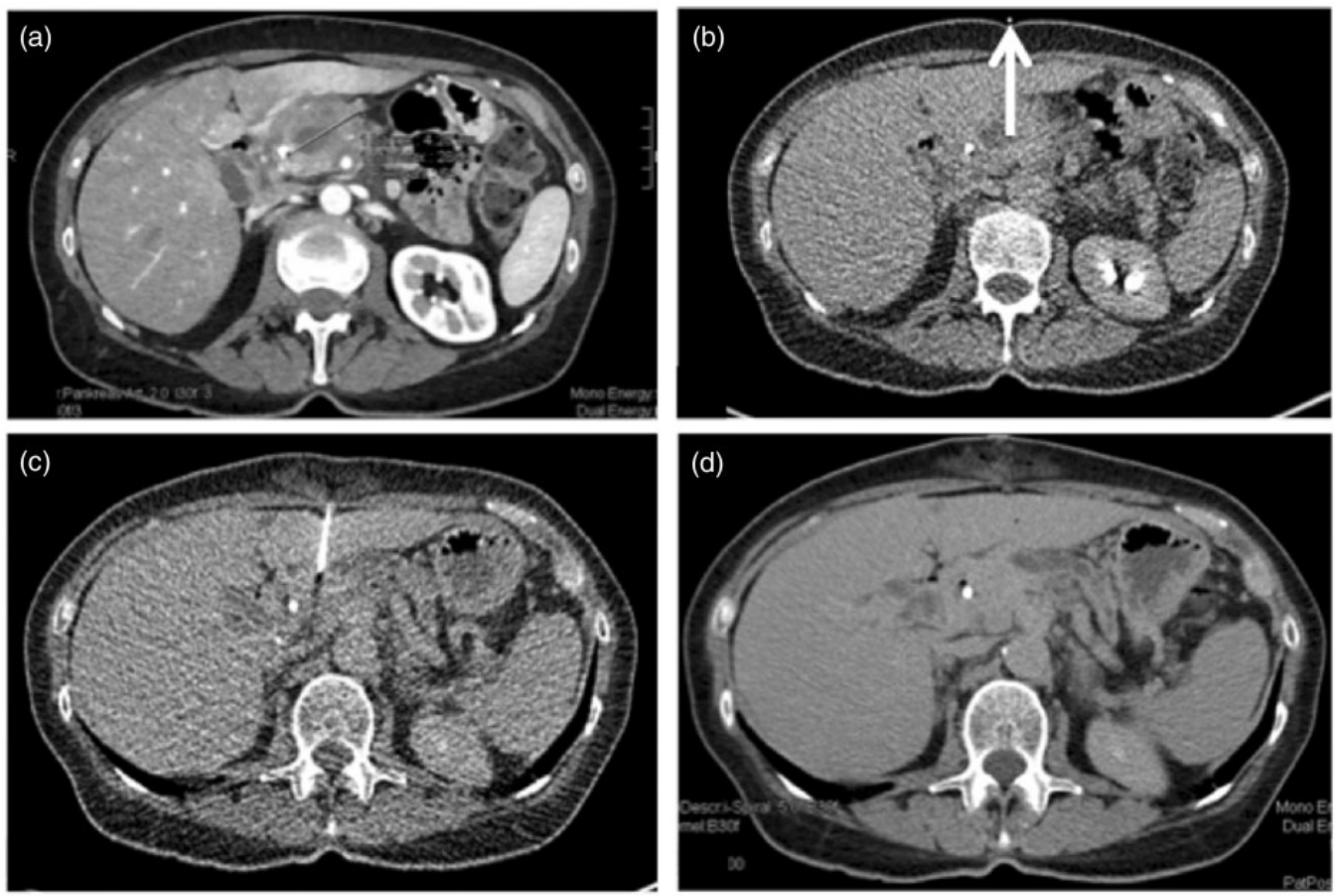

Fig. I. CT images of a 69-year-old female patient with an unclear lesion in the pancreatic head; the histological result revealed a G3 adenocarcinoma. (a) Arterial phase of pre-interventional planning scan. (b) Identification of the needle insertion point under CT fluoroscopic control, by the help of the local anesthesia needle. The white arrow points to the needle. (c) True-cut biopsy under CT fluoroscopic control by a transhepatic pathway. (d) Non-enhanced post-interventional control scan. 
dose by the adjusted formula for CT fluoroscopy: $\mathrm{E}=\mathrm{DLP}{ }^{*} \mathrm{k}(\mathrm{k}=0.018)$ according to Spelsberg et al. (18).

\section{Complications}

To assess intra-interventional complications, an interventional radiologist with more than 10 years of experience examined all CT images of the procedures. CT scans were evaluated regarding the needle access trajectory, bleeding, and affected organs. For evaluation of complications during the 30-day post-interventional period, patient files were reviewed. Complications were divided into major and minor according to Gupta et al. (19).

\section{Statistical analysis}

For data collection and statistical analysis, the software SPSS Version 17.0 (SPSS Inc., Chicago, IL, USA) was used.

\section{Results}

\section{Patient characteristics}

One hundred and one consecutive patients (54 women, 47 men) with an unclear pancreatic lesion received a total of 104 percutaneous CT fluoroscopic-guided true-cut pancreatic biopsies. Mean age at the intervention was $63.9 \pm 12.6$ years (age range, $17-90$ years). Mean diameter of the pancreatic lesions in the axial CT slice was $4.7 \pm 2.3 \mathrm{~cm}$ (range, $1.5-13 \mathrm{~cm}$ ). Twentynine $(28.7 \%)$ patients had undergone an ERCP or EUS-FNA with unsuccessful tissue sampling before the CT intervention. Localization of the lesions and patient characteristics are summarized in Table 1.

\section{Intervention characteristics}

One hundred and four interventions were retrospectively analyzed. Access path was ventral $(n=60)$, dorsal $(\mathrm{n}=20)$, left lateral $(\mathrm{n}=11)$, and right lateral $(\mathrm{n}=11)$. In 31 cases $(30.4 \%)$, an access path through neighboring organs (liver, stomach, and colon) was chosen. In case of colonic transgression, an antibiotic with piperacillin/tazobactam was prophylactically administered pre- and intra-interventionally. In two cases a transpulmonal pathway was chosen, one of them intentionally through the lower lobe and the other one unintentionally through the left pleural recess. In both cases neither a pneumothorax nor other pulmonary complication was seen. In the remaining $71(69.6 \%)$ cases a direct access was feasible. Mean
Table I. Patient characteristics.

\begin{tabular}{|c|c|c|}
\hline Variable & $\begin{array}{l}\text { Absolute } \\
\text { numbers } \\
\text { (frequency) }\end{array}$ & $\begin{array}{l}\text { Mean value } \pm S D \\
\text { (range) }\end{array}$ \\
\hline Patients (n) & 101 (100\%) & \\
\hline \multicolumn{3}{|l|}{ Gender } \\
\hline - Female & 54 (54.5\%) & \\
\hline - Male & 47 (45.5\%) & \\
\hline Age (years) & & $\begin{array}{l}63.5 \pm 12.1 \\
(17-90)\end{array}$ \\
\hline Lesion size $(\mathrm{cm})$ & & $\begin{array}{l}4.7 \pm 2.3 \\
(1.5-13)\end{array}$ \\
\hline \multicolumn{3}{|l|}{ Localization } \\
\hline - Head & 48 (47.5\%) & \\
\hline - Head/Body & $8(7.9 \%)$ & \\
\hline - Body & $18(17.8 \%)$ & \\
\hline - Body/Tail & $5(5.0 \%)$ & \\
\hline - Tail & $13(12.9 \%)$ & \\
\hline - Uncinate process & $5(5.0 \%)$ & \\
\hline - Entire pancreas & $4(4.0 \%)$ & \\
\hline $\begin{array}{l}\text { Patients with } \\
\text { pre-interventional ERCP } \\
\text { or EUS- FNA for } \\
\text { unsuccessful sampling }\end{array}$ & 29 (28.7\%) & \\
\hline - EUS-FNA & $2 \mathrm{I}(20.8 \%)$ & \\
\hline - ERCP & $8(7.9 \%)$ & \\
\hline
\end{tabular}

ERCP, endoscopic retrograde cholangiopancreatography; EUS-FNA, endoscopic ultrasound with fine-needle aspiration.

DLP was $810 \pm 425 \mathrm{mGy}^{*} \mathrm{~cm}$, including the planning and control CT scan (mean DLP $736 \pm 391 \mathrm{mGy}^{*} \mathrm{~cm}$ ) and the intra-interventional CT fluoroscopy images (mean DLP $74 \pm 72 \mathrm{mGy} * \mathrm{~cm}$ ). Therefore, $89.9 \%$ of effective patient radiation dose was caused by the planning and post-interventional control CT scan and $10.1 \%$ by intra-interventional CT fluoroscopy.

Mean effective patient dose was $12.5 \pm 6.5 \mathrm{mSv}$, including effective doses of pre-interventional planning scan, intra-interventional CT fluoroscopy, and postinterventional control scan. The intervention characteristics are summed up in Table 2.

\section{Outcome and complications}

Ninety-eight of 104 interventions (94.2\%) were technically successful and a true-cut biopsy could be obtained. In six cases $(5.8 \%)$ the intervention had to be stopped without a successful biopsy, three of them due to intra-interventional complications (see below). In the remaining cases reasons were: incompliant patient $(n=1)$, software problems with the CT scanner 
Table 2. Intervention characteristics.

\begin{tabular}{|c|c|c|c|}
\hline Variable & $\begin{array}{l}\text { Absolute numbers } \\
\text { (frequency) }\end{array}$ & Mean value \pm SD (range) & Mode (range) \\
\hline \multicolumn{4}{|l|}{ Access path } \\
\hline - Ventral & $60(59.0 \%)$ & & \\
\hline - Dorsal & 20 (19.6\%) & & \\
\hline - Left lateral & II (10.8\%) & & \\
\hline - Right lateral & II (10.8\%) & & \\
\hline \multicolumn{4}{|l|}{ Access path through } \\
\hline - Liver & $17(16.7 \%)$ & & \\
\hline - Stomach & 7 (6.9\%) & & \\
\hline - Colon & $5(4.9 \%)$ & & \\
\hline - Lung / Pleural & $2(2.0 \%)$ & & \\
\hline Needle length (mode) $(\mathrm{cm})$ & & & $13(10-20)$ \\
\hline Needle diameter (mode) (G) & & & $18(13-18)$ \\
\hline Intracorporal length of needle trajectory $(\mathrm{cm})$ & & $9.6 \pm 3.1 \mathrm{~cm}(3.4-17.7)$ & \\
\hline Number of samples (mode) & & & $I(I-5)$ \\
\hline Duration of intervention (min) & & $27.5 \pm 8(|4-5|)$ & \\
\hline Total effective patient radiation dose (mSv) & & $12.5 \pm 6.5(2.0-46.6)$ & \\
\hline
\end{tabular}

$(\mathrm{n}=1)$, decision not to perform biopsy due to safety reasons after evaluation of the planning CT (target area too close to SMA) $(n=1)$. The primarily unsuccessful interventions could not be attributed to a particular intervention setting (access path, penetrated organs, length or needle diameter).

Eighty-eight of the 98 technically successful samples led to a definitive pathological diagnosis, consistent with clinical success $(89.8 \%)$. In the remaining 10 cases no definitive pathological diagnosis of the lesion could be obtained. The histopathological results of our patient cohort are listed in Table 3. Three patients with prior clinically unsuccessful biopsy received another CTguided biopsy, which then led to a successful tissue sampling. The other patients with unsuccessful biopsies received an ERCP-guided biopsy without successful tissue sampling $(n=1)$, open surgical biopsy with successful tissue sampling $(n=4)$, or no further biopsy $(n=5)$.

Eighty-two of 104 biopsies (78.9\%) were performed without complications. In 19 of the remaining cases, minor complications occurred. All cases were minor self-limiting hematomas evident in the control scan. None of these complications led to clinical consequences or a longer hospital stay. Additionally, no case of tumor needle tract seeding was observed during the 60-day follow-up.

In three patients, major complications related to the CT-guided intervention occurred:

Patient A (F, 75 years, needle diameter 18 G): Immediately after needle positioning in the pancreatic head (lesion diameter of $3.1 \mathrm{~cm}$ ), a growing opacity of the mesenteric fat tissue was visible under CT fluoroscopy. After interruption of the intervention a biphasic contrast-enhanced CT scan showed a definite bleeding. The patient was immediately brought to the angiography unit where no active bleeding was found. The patient was moved to the ICU due to a hemoglobin value drop from $14.1 \mathrm{~g} / \mathrm{dl}$ to $10 \mathrm{~g} / \mathrm{dl}$ and packed red blood cells (PRBC) had to be substituted. One month after the puncture the patient underwent a successful Whipple operation. Intraoperatively, a residual hematoma $(10 \times 6 \mathrm{~cm})$ was found.

Patient B (M, 64 years, needle diameter 18 G) (Fig. 2): After two samples were successfully taken out of a lesion $(1.5 \mathrm{~cm})$ of the pancreatic head, the post-interventional control CT scan showed peripancreatic bleeding. The position of the bleeding was subhepatic extending to the abdominal aortic bifurcation. CT angiography was immediately performed without detecting the origin of the bleeding. Maximum size of the hematoma was $19 \times 10 \mathrm{~cm}$. Eight PRBCs had to be administered and during the following emergency operation the underlying bleeding of the epiploic artery could be controlled.

Patient C (F, 63 years, needle diameter $16 \mathrm{G})$ : Three samples could be taken successfully out of the pancreatic head lesion $(5.3 \mathrm{~cm})$. The control CT revealed an arterial bleeding. In the angiography unit an extravasation out of a side branch of the gastroduodenal artery (GDA) was detected. Bleeding could be stopped by selective microcoil embolization (Fig. 2). 
Table 3. Histologic results.

\begin{tabular}{lc}
\hline & $\mathrm{n}(98)$ \\
\hline Definitive histopathologic diagnosis & $88(89.8 \%)$ \\
Adenocarcinoma & $63(64.3 \%)$ \\
Acinar cell carcinoma & $\mathrm{I}(1 \%)$ \\
Hepatocellular carcinoma & $\mathrm{I}(1 \%)$ \\
Cystadenoma & $2(2 \%)$ \\
Neuroendocrine tumor & $6(6.1 \%)$ \\
Pancreatitis & $6(6.1 \%)$ \\
Lymphoma & $5(5.1 \%)$ \\
Metastases of other tumors & $4(4.1 \%)$ \\
No definitive histopathologic & $10(10.2 \%)$ \\
diagnosis (normal pancreatic tissue) &
\end{tabular}

No correlation between needle diameter and complications was found.

\section{Post-procedural morbidity and mortality}

Due to fast tumor progression, many of the patients died weeks or months after the diagnosis and the intervention. In the 30-day post-procedural period no patient had other complications than mentioned above or died attributable to the intervention.

\section{Discussion}

In this study technical and clinical outcome of all CT fluoroscopy-guided pancreatic true-cut biopsies performed during a 10-year period at our center were
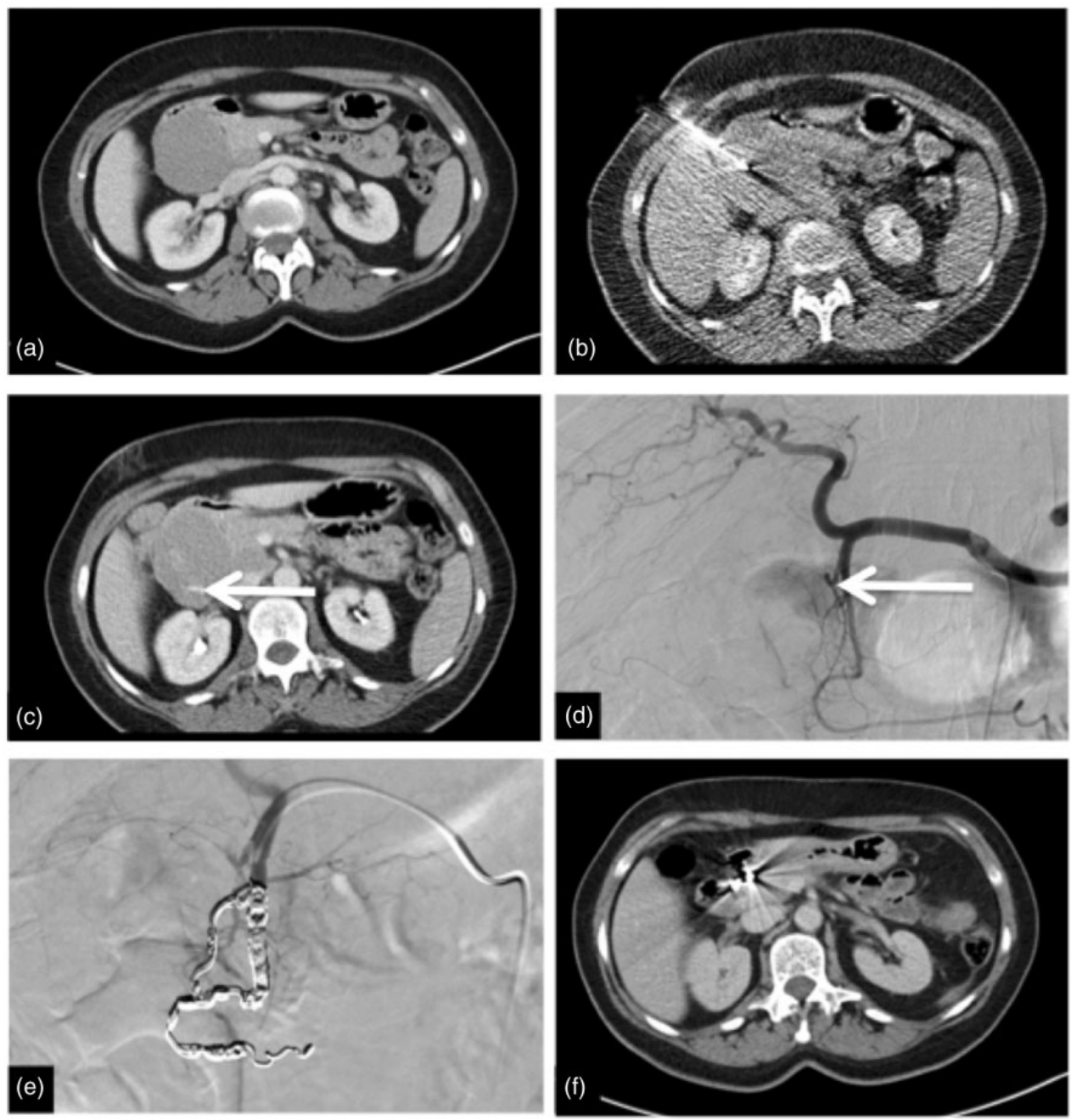

Fig. 2. Images of a 63-year-old woman with an unclear lesion in the pancreatic head. Histological result revealed a mantle cell lymphoma. (a) Portal-venous phase of pre-interventional planning scan. (b) True-cut biopsy under CT fluoroscopic control with a I6 G $13 \mathrm{~cm}$ biopsy system. (c) Contrast-enhanced control scan, the white arrow points on an active arterial bleeding. (d) Angiography, the white arrow points on an active bleeding from the gastroduodenal artery. (e) Successful embolization with microcoils. (f) Control scan. 
investigated. A total of 104 interventions were evaluated; in 88 cases $(87 \%)$ a definitive histopathological result and diagnosis could be achieved. Six cases were technically unsuccessful and in ten cases no final diagnosis could be established. Most complications did not imply any clinical consequences for the patients. In three cases, major bleeding complications occurred requiring further treatment.

The high technical and clinical success rates underline the high reliability of this procedure.

The clinical importance of this procedure is shown by the fact that $29(28.7 \%)$ of our patients underwent CT fluoroscopy-guided intervention after an unsuccessful ERCP-biopsy (8 cases) or EUS-FNA (21 cases). Otherwise an explorative surgery was the only other option to obtain a diagnostic specimen.

Former studies about EUS-FNA of pancreatic lesions have shown an accuracy of $78-95 \%$ with a major complication rate of $1.97-2.53 \%$ (20). An advantage of EUS-FNA compared to CT-guided interventions is the missing radiation exposure to patient and IR $(20,21)$. However, if the tumor is small or located in the pancreatic body or tail, performing EUS-FNA might be problematic. Another disadvantage of ultrasound guidance is the poor image quality due to possible intestinal gas superposition and the required sedation.

ERCP is a diagnostic and a therapeutic procedure (22). However, the reported accuracy of ERCP-guided pancreatic biopsies is quite low $(46.7 \%)$ (7). Furthermore, the complication rate is higher compared to other procedures $(15.9 \%)$ (23). The main complication of ERCP is post-interventional pancreatitis (5\% of the interventions) which is a severe condition in these patients and rarely might even be fatal (24). Additionally, general anesthesia is often required for complex ERCP procedures.

CT fluoroscopic guidance is characterized by a high success rate and the potential to reach every pancreatic region for biopsy. Even an indirect access path traversing other organs can be chosen without severe clinical consequences. Major vessels covering the lesion can represent the main clinical limitation. According to the literature the risk of post-interventional tumor seeding and peritoneal carcinomatosis is not significant in all three types of procedures (25), and tumor seeding was not observed in our patient cohort correspondingly.

A large number of studies have evaluated the value of interventional CT fluoroscopy guidance in various anatomic regions (14). Nevertheless, to the best of our knowledge this is the largest patient series exclusively undergoing CT fluoroscopy-guided pancreatic true-cut biopsies. Main advantages of CT fluoroscopy are the shorter procedure time and lower radiation dose compared to sequential CT guidance (15).
Regarding accuracy, technical and clinical outcome, our study is in line with other similar studies focusing on CT-guided pancreatic core biopsies $(9,10)$. The complication rate of CT-guided pancreatic biopsy was $8.7 \%$ reported by Tyng et al. (8) and $2.8 \%$ reported by Paulsen et al. (9), respectively. Our higher complication rate $(21.1 \%)$ might be explained by the fact that small hematomas of no clinical relevance were reported as minor complications (19). Tyng et al. reported a success rate of $98.1 \%$ (8); in addition, Paulsen et al. reported a $94.4 \%$ success rate (9); our slightly lower rate of $89.8 \%$ can be explained by the fact that in our study the intervention was only regarded as clinical successful if not only tissue sampling but also a definitive histological result was reached, reflecting the most significant result for the treatment strategy. Of note, only 63 out of 88 patients with a definitive histological result were diagnosed with pancreatic adenocarcinoma (Table 3); the remaining 25 patients had a wide spectrum of other pathological diagnoses highlighting the need for histological confirmation before initiating chemotherapy for suspected pancreatic cancer.

The main clinical relevant complication of pancreatic biopsies is active arterial bleeding. Therefore, prior to the intervention a biphasic planning $\mathrm{CT}$ is mandatory to carefully evaluate the needle access path and the localization of vessels at risk. A post-interventional CT scan is equally necessary to detect early complications. Basically in a straightforward intervention an unenhanced control scan is sufficient to rule out severe complications. In addition, the procedure has to be performed by an experienced interventional radiologist to assure a high quality of the biopsy including a lower radiation dose compared with non-experienced interventional radiologists. In this study, up to six different experienced board-certified interventional radiologist's with a minimum of 10 years of experience or a radiology resident in the third year up to fifth year under the supervision of a board-certified radiologist carried out the intervention. Equally, a multiprofessional backup team consisting of surgery, anesthesiology, and intensive care units is essential in case of major complications (19).

The main limitation of this study is that all data are based on a retrospective single-center analysis. However, as there are clear data that support improved treatment outcomes for patients with pancreatic cancer treated at high-volume centers, also the pre-therapeutic diagnostic procedures should - at least in our opinion-be performed at such specialized cancer centers with an experienced team of interventional radiologists.

In conclusion, we showed that CT fluoroscopyguided pancreatic true-cut biopsy is effective, safe, and minimally invasive and therefore a reliable alternative to endosonographic, endoscopic, or surgical tissue 
sampling. This procedure might allow obtaining definitive diagnosis early and thereby shortening the time to treatment initiation in patients with pancreatic malignancies. Further prospective studies have to define the exact role and indications for CT-guided true-cut biopsy compared to alternative invasive, imageguided, and endoscopic methods.

\section{Declaration of Conflicting Interests}

The author(s) declared no potential conflicts of interest with respect to the research, authorship, and/or publication of this article.

\section{Funding}

The author(s) received no financial support for the research, authorship, and/or publication of this article.

\section{References}

1. Sener SF, Fremgen A, Menck HR, et al. Pancreatic cancer: a report of treatment and survival trends for 100,313 patients diagnosed from 1985-1995, using the National Cancer Database. J Am Coll Surg 1999;189:1-7.

2. Hidalgo M. Pancreatic cancer. N Engl J Med 2010;362: 1605-1617.

3. Clarke DL, Clarke BA, Thomson SR, et al. The role of preoperative biopsy in pancreatic cancer. HPB (Oxford) 2004;6:144-153.

4. Kamisawa T, Wood LD, Itoi T, et al. Pancreatic cancer. Lancet 2016;388:73-85.

5. Conroy T, Bachet JB, Ayav A, et al. Current standards and new innovative approaches for treatment of pancreatic cancer. Eur J Cancer 2016;57:10-22.

6. Goldin SB, Bradner MW, Zervos EE, et al. Assessment of pancreatic neoplasms: review of biopsy techniques. J Gastrointest Surg 2007;11:783-790.

7. Wakatsuki T, Irisawa A, Bhutani MS, et al. Comparative study of diagnostic value of cytologic sampling by endoscopic ultrasonography-guided fine-needle aspiration and that by endoscopic retrograde pancreatography for the management of pancreatic mass without biliary stricture. J Gastroenterol Hepatol 2005;20:1707-1711.

8. Li H, Hu Z, Chen J, et al. Comparison of ERCP, EUS, and ERCP combined with EUS in diagnosing pancreatic neoplasms: a systematic review and meta-analysis. Tumour Biol 2014;35:8867-8874.

9. Tyng CJ, Almeida MF, Barbosa PN, et al. Computed tomography-guided percutaneous core needle biopsy in pancreatic tumor diagnosis. World $\mathrm{J}$ Gastroenterol 2015;21:3579-3586.

10. Paulsen SD, Nghiem HV, Negussie E, et al. Evaluation of imaging-guided core biopsy of pancreatic masses. Am J Roentgenol 2006;187:769-772.

11. Amin Z, Theis B, Russell RC, et al. Diagnosing pancreatic cancer: the role of percutaneous biopsy and CT. Clin Radiol 2006;61:996-1002.
12. Hsu MY, Pan KT, Chen CM, et al. CT-guided percutaneous core-needle biopsy of pancreatic masses: comparison of the standard mesenteric/retroperitoneal versus the trans-organ approaches. Clin Radiol 2016;71:507-512.

13. Zech CJ, Helmberger T, Wichmann MW, et al. Large core biopsy of the pancreas under CT fluoroscopy control: results and complications. J Comput Assist Tomogr 2002;26:743-749.

14. Paprottka PM, Helmberger T, Reiser MF, et al. [Computed tomography guidance: fluoroscopy and more]. Radiologe 2013;53:974-985.

15. Sarti M, Brehmer WP, Gay SB. Low-dose techniques in CT-guided interventions. Radiographics 2012;32: 1109-1119.

16. Patel IJ, Davidson JC, Nikolic B, et al. Consensus guidelines for periprocedural management of coagulation status and hemostasis risk in percutaneous imageguided interventions. J Vasc Interv Radiol 2012;23: 727-736.

17. Deak PD, Smal Y, Kalender WA. Multisection CT protocols: sex- and age-specific conversion factors used to determine effective dose from dose-length product. Radiology 2010;257:158-166.

18. Spelsberg FW, Hoffmann RT, Lang RA, et al. CT fluoroscopy guided percutaneous gastrostomy or jejunostomy without (CT-PG/PJ) or with simultaneous endoscopy (CT-PEG/PEJ) in otherwise untreatable patients. Surg Endosc 2013;27:1186-1195.

19. Gupta S, Wallace MJ, Cardella JF, et al. Quality improvement guidelines for percutaneous needle biopsy. J Vasc Interv Radiol 2010;21:969-975.

20. Yoshinaga S, Suzuki H, Oda I, et al. Role of endoscopic ultrasound-guided fine needle aspiration (EUS-FNA) for diagnosis of solid pancreatic masses. Dig Endosc 2011; 23(Suppl. 1): 29-33.

21. Lewitowicz P, Matykiewicz J, Heciak J, et al. Percutaneous fine needle biopsy in pancreatic tumors: a study of 42 cases. Gastroenterol Res Pract 2012;2012: 908963.

22. Suissa A, Yassin K, Lavy A, et al. Outcome and early complications of ERCP: a prospective single center study. Hepatogastroenterology 2005;52:352-355.

23. Christensen M, Matzen P, Schulze S, et al. Complications of ERCP: a prospective study. Gastrointest Endosc 2004; 60:721-731.

24. Andriulli A, Loperfido S, Napolitano G, et al. Incidence rates of post-ERCP complications: a systematic survey of prospective studies. Am J Gastroenterol 2007;102: 1781-1788.

25. Ikezawa K, Uehara H, Sakai A, et al. Risk of peritoneal carcinomatosis by endoscopic ultrasound-guided fine needle aspiration for pancreatic cancer. J Gastroenterol 2013;48:966-972. 